VOL. 66 (2002) [405-418]

\title{
ASYMPTOTICAL SMOOTHNESS AND ITS APPLICATIONS
}

\author{
Wieslawa Kaczor and Stanislaw Prus
}

In this paper we introduce the notion of asymptotical smoothness of a Banach space and show that it is strongly related to the Kadec-Klee property. This notion is then applied to obtain new theorems about weak convergence of almost orbits of three various types of semigroups of mappings.

\section{INTRODUCTION}

Geometrical properties of Banach spaces play crucial role in many problems of metric fixed point theory. This includes the Kadec-Klee property, the Opial property, and their various modifications (see [23]). Actually, some authors attribute the first of these properties to M. Radon and F. Riesz (see [2, p. 133]), so they call it the Radon-Riesz property. It is also called property $(\mathrm{H})$ (see [5, p. 112]). The idea of the Opial property comes from [21].

These properties are defined in terms of weakly convergent sequences. However, in some applications we deal with nets. According to the terminology of [4], the counterpart of the Kadec-Klee property for nets is called the Kadec property.

In some papers concerning nonlinear ergodic theory, to get weak convergence of suitable sequences or nets in a Banach space $X$, authors use the Kadec-Klee property and the Kadec property (respectively) of the dual space $X^{*}$ (see, for example, [7] and $[10,11,12])$. In the next section of our paper we introduce the notion of asymptotical smoothness and prove that it is partially dual to the Kadec property.

In other papers dealing with ergodic theory, a Banach space $X$ is assumed to have either the Opial property for sequences or the Opial property for nets [16]. This leads to the general problem: under what assumptions one can replace sequences by nets in a given condition concerning weakly convergent sequences. A solution of this problem for the Kadec-Klee property can be found in [14] (see also [1, p. 113]). In this paper we find a more general result in this direction. It shows in particular that if $X$ is reflexive, then the Opial property for sequences is equivalent to the Opial property for nets.

The last section of our paper is devoted to the study of weak convergence of almost orbits of three types of right reversible semitopological semigroups in uniformly convex

Received 6th March, 2002

Research supported by the KBN grant 2P03A02915.

Copyright Clearance Centre, Inc. Serial-fee code: 0004-9727/02 \$A2.00+0.00. 
and asymptotically smooth Banach spaces. Namely, we consider nonexpansive, asymptotically nonexpansive commutative and asymptotically nonexpansive-type semigroups.

\section{ASYMPTOTICALLY SMOOTH SPACES}

In this paper we shall consider only infinite dimensional real Banach spaces. However, our results hold also for complex spaces. This case requires only minor modifications of some proofs. Let $X$ be a Banach space. Its closed unit ball and the unit sphere will be denoted by $B_{X}$ and $S_{X}$, respectively. By $\mathcal{B}$ we denote the family of all closed subspaces of $X$ with finite codimension. Given $t \geqslant 0$ and $x \in S_{X}$, we put

$$
\delta(t ; x)=\inf _{E \in \mathcal{B}} \sup _{y \in S_{E}}\|x+t y\|-1 .
$$

This coefficient was introduced in [19]. It is easy to see that $t \mapsto \delta(t ; x)$ is a continuous nonnegative function such that $\delta(t ; x) / t$ is nondecreasing.

DEFINITION 2.1: We say that the space $X$ is asymptotically smooth if

$$
\lim _{t \rightarrow 0} \frac{\delta(t ; x)}{t}=0
$$

for every $x \in S_{X}$.

It is worth while to mention here that for some spaces the coefficient $\delta(t ; x)$ can be described in explicit terms $([\mathbf{1 9}])$. Namely, $\delta(t ; x)=\left(1+t^{p}\right)^{1 / p}-1, t \geqslant 0$, if $X=l_{p}$, or more generally, if $X=\left(\sum_{n=1}^{\infty} X_{n}\right)_{l_{p}}$ where $1 \leqslant p<\infty$ and $\left(X_{n}\right)$ is a sequence of finite dimensional spaces. Moreover, if $X=c_{0}$, then $\delta(t ; x)=0$ for $0 \leqslant t<1$ and $\delta(t ; x)=t-1$ for $t \geqslant 1$. The same formulae hold for the space $X=\left(\sum_{n=1}^{\infty} X_{n}\right)_{c_{0}}$ where $\left(X_{n}\right)$ is a sequence of finite dimensional spaces. Thus $c_{0}$ and $l_{p}$ with $p \neq 1$ are asymptotically smooth but $l_{1}$ lacks this property.

To state our first result, recall that the norm of $X$ is said to be Fréchet differentiable (see, for example, [23]) if for every $x \in S_{X}$,

$$
\lim _{t \rightarrow 0} \sup _{y \in S_{X}}\left|\frac{1}{t}(\|x+t y\|-\|x\|)-\phi_{x}(y)\right|=0,
$$

where $\phi_{x} \in X^{*}$ is the Gateaux derivative of the norm at $x$.

PROPOS ITION 2.1. If the norm of $X$ is Fréchet differentiable, then $X$ is asymptotically smooth.

Proof: Given $x \in S_{X}$, we take $x^{*} \in X^{*}$ such that $x^{*}(x)=1=\left\|x^{*}\right\|$ and put $E_{0}=\operatorname{ker} x^{*}$. Then $\|x+t y\| \geqslant x^{*}(x+t y)=1$ if $y \in E_{0}$. This shows that $\phi_{x}(y) \geqslant 0$. Consequently, $\phi_{x}(y)=0$ for every $y \in E_{0}$. It follows that

$$
\sup _{y \in S_{X}}\left|\frac{1}{t}(\|x+t y\|-1)-\phi_{x}(y)\right| \geqslant \sup _{y \in S_{E_{0}}} \frac{1}{t}(\|x+t y\|-1) \geqslant \frac{1}{t} \delta(t ; x)
$$


for every $t>0$, and since the norm of $X$ is Fréchet differentiable, we see that $\lim _{t \rightarrow 0} \delta(t ; x) / t$ $=0$.

On the other hand, $c_{0}$ is asymptotically smooth while its norm is not even Gateaux differentiable.

Asymptotical smoothness can be characterised in terms of nets. Given a Banach space $X, x \in S_{X}$ and $t \geqslant 0$, we put

$$
\delta_{0}(t ; x)=\sup \left\{\limsup _{\alpha \in A}\left\|x+t x_{\alpha}\right\|-1\right\}
$$

where the supremum is taken over all weakly null nets $\left(x_{\alpha}\right)_{\alpha \in A}$ in $B_{X}$ and

$$
\delta_{1}(t ; x)=\sup \left\{\liminf _{\beta \in A} \liminf _{\alpha \in A}\left\|x+t\left(x_{\alpha}-x_{\beta}\right)\right\|-1\right\}
$$

where the supremum is taken over all nets $\left(x_{\alpha}\right)_{\alpha \in A}$ in $B_{X}$.

THEOREM 2.2. Let $X$ be a Banach space, $x \in S_{X}$ and $t \geqslant 0$. Then

$$
\delta_{0}(t ; x)=\delta(t ; x)
$$

and

$$
\delta(t ; x) \leqslant \delta_{1}(t ; x) \leqslant \delta(2 t ; x)
$$

Proof: We first show that $\delta(t ; x) \leqslant \delta_{0}(t ; x)$. To this end we consider the set $\mathcal{B}$ with the order given as follows: $E_{1} \leqslant E_{2}$ if $E_{2} \subset E_{1}$ where $E_{1}, E_{2} \in \mathcal{B}$. Given $\varepsilon>0$, for each $E \in \mathcal{B}$ we find $y_{E} \in S_{E}$ so that

$$
\left\|x+t y_{E}\right\|-1>\delta(t ; x)-\varepsilon
$$

It is easy to see that the net $\left(y_{E}\right)_{E \in \mathcal{B}}$ converges weakly to 0 . Therefore,

$$
\delta(t ; x)-\varepsilon \leqslant \limsup _{E \in \mathcal{B}}\left\|x+t y_{E}\right\|-1 \leqslant \delta_{0}(t ; x)
$$

Since $\varepsilon>0$ is arbitrary, we get the desired inequality.

Let now $\left(x_{\alpha}\right)_{\alpha \in A}$ be a weakly null net in $B_{X}$. Then

$$
\lim _{\alpha \in A}\left\|x+t x_{\alpha}\right\| \leqslant \liminf _{\alpha \in A} \liminf _{\beta \in A}\left\|x+t\left(x_{\alpha}-x_{\beta}\right)\right\| \leqslant \delta_{1}(t ; x)+1
$$

provided that the limit on the left hand side exists. This shows that $\delta_{0}(t ; x) \leqslant \delta_{1}(t ; x)$, which in view of the first part of the proof gives $\delta(t ; x) \leqslant \delta_{1}(t ; x)$.

Our next aim is to prove that $\delta_{1}(t ; x) \leqslant \delta(2 t ; x)$. Given $\varepsilon>0$, we find a subspace $E \in \mathcal{B}$ so that $\|x+2 t y\|-1 \leqslant \delta(2 t ; x)+\varepsilon$ for every $y \in S_{E}$. Let us put $m=\operatorname{codim} E$. There are elements $z_{1}, \ldots, z_{m} \in S_{X}$ and functionals $z_{1}^{*}, \ldots, z_{m}^{*} \in X^{*}$ such that $E=\bigcap_{i=1}^{m} \operatorname{ker} z_{i}^{*}$, 
$z_{k}^{*}\left(z_{k}\right)=1$ and $z_{k}^{*}\left(z_{i}\right)=0$ for every $i=1, \ldots, m$ and $k \neq i$. Given a weakly null net $\left(x_{\alpha}\right)_{\alpha \in A}$ in $B_{X}$, we set

$$
y_{\alpha}=x_{\alpha}-\sum_{i=1}^{m} z_{i}^{*}\left(x_{\alpha}\right) z_{i} .
$$

Then $z_{k}^{*}\left(y_{\alpha}\right)=0$ for $k=1, \ldots, m$, which shows that $y_{\alpha} \in E$. Moreover, $\left\|y_{\alpha}-y_{\beta}\right\|$ $\leqslant\left\|x_{\alpha}-x_{\beta}\right\|+d_{\alpha \beta} \leqslant 2+d_{\alpha \beta}$ where $d_{\alpha \beta}=\sum_{i=1}^{m}\left|z_{i}^{*}\left(x_{\alpha}\right)-z_{i}^{*}\left(x_{\beta}\right)\right|$. It follows that

$$
\begin{aligned}
\frac{1}{t}\left(\left\|x+t\left(y_{\alpha}-y_{\beta}\right)\right\|-1\right) & \leqslant \frac{\left\|y_{\alpha}-y_{\beta}\right\|}{t\left(2+d_{\alpha \beta}\right)}\left(\left\|x+\frac{t\left(2+d_{\alpha \beta}\right)}{\left\|y_{\alpha}-y_{\beta}\right\|}\left(y_{\alpha}-y_{\beta}\right)\right\|-1\right) \\
& \leqslant \frac{\left\|y_{\alpha}-y_{\beta}\right\|}{t\left(2+d_{\alpha \beta}\right)}\left(\left\|x+\frac{2 t}{\left\|y_{\alpha}-y_{\beta}\right\|}\left(y_{\alpha}-y_{\beta}\right)\right\|-1+t d_{\alpha \beta}\right) \\
& \leqslant \frac{\left\|y_{\alpha}-y_{\beta}\right\|}{t\left(2+d_{\alpha \beta}\right)}\left(\delta(2 t ; x)+\varepsilon+t d_{\alpha \beta}\right)
\end{aligned}
$$

whenever $y_{\alpha} \neq y_{\beta}$. Hence

$$
\left\|x+t\left(y_{\alpha}-y_{\beta}\right)\right\|-1 \leqslant \delta(2 t ; x)+\varepsilon+t d_{\alpha \beta},
$$

which holds also in the case when $y_{\alpha}=y_{\beta}$. Consequently,

$$
\left\|x+t\left(x_{\alpha}-x_{\beta}\right)\right\|-1 \leqslant\left\|x+t\left(y_{\alpha}-y_{\beta}\right)\right\|-1+t d_{\alpha \beta} \leqslant \delta(2 t ; x)+\varepsilon+2 t d_{\alpha \beta}
$$

for all $\alpha, \beta \in A$. Passing to a subnet, we can assume that $\lim _{\alpha \in A} z_{i}^{*}\left(x_{\alpha}\right)$ exists for $i$ $=1, \ldots, m$. Then $\lim _{\beta \in A} \lim _{\alpha \in A} d_{\alpha \beta}=0$. This shows that

$$
\liminf _{\beta \in A} \liminf _{\alpha \in A}\left\|x+t\left(x_{\alpha}-x_{\beta}\right)\right\|-1 \leqslant \delta(2 t ; x)+\varepsilon .
$$

It follows that $\delta_{1}(t ; x) \leqslant \delta(2 t ; x)+\varepsilon$. Passing to the limit with $\varepsilon \rightarrow 0$, we obtain $\delta_{1}(t ; x) \leqslant \delta(2 t ; x)$. A straightforward modification of the last part of the proof gives the inequality $\delta_{0}(t ; x) \leqslant \delta(t ; x)$, which concludes the proof.

Recall that the James space $J$ is defined as the space of all real sequences $x=\left(x_{n}\right)$ converging to 0 such that

$$
\|x\|=\sup \left(\sum_{k=1}^{m-1}\left|x_{n_{k+1}}-x_{n_{k}}\right|^{2}+\left|x_{n_{m}}-x_{n_{1}}\right|^{2}\right)^{1 / 2}<\infty,
$$

where the supremum is taken over all sequences of positive integers $n_{1}<n_{2}<\ldots$ $<n_{m}$ (see [8]). In [19], it is mentioned that in this case $\delta(t ; x)=\left(1+t^{2}\right)^{1 / 2}-1$ for all $x \in S_{J}$ and $t \geqslant 0$, which is not true. Indeed, consider the sequence of vectors $e_{n}=(0, \ldots, 0,1,0, \ldots) / \sqrt{2}$, where 1 is the $n$th coordinate. Clearly, $\left(e_{n}\right)$ is weakly null, $\left\|e_{n}\right\|=1$ and $\left\|e_{1}-t e_{n}\right\|=1+t$ for every $n$ and $t \geqslant 0$. In view of Theorem 2.2 this shows that $\delta\left(t ; e_{1}\right)=t$. Consequently, $J$ is not asymptotically smooth. It may however 
be renormed to have this property. The appropriate norm was defined in [22]. Namely, given $x=\left(x_{n}\right) \in J$, we put

$$
\|x\|_{1}=\sup \left(\sum_{k=1}^{m}\left|x_{n_{2 k-1}}-x_{n_{2 k}}\right|^{2}+2\left|x_{n_{2 m+1}}\right|^{2}\right)^{1 / 2},
$$

where the supremum is taken over all sequences of positive integers $n_{1}<n_{2}<\ldots$ $<n_{2 m+1}$. Then $\|x\| / \sqrt{3} \leqslant\|x\|_{1} \leqslant 2\|x\|$ for every $x \in J$, so the norm $\|\cdot\|_{1}$ is equivalent to $\|\cdot\|$. Moreover, if $x \in \operatorname{span}\left\{e_{k}\right\}_{k=1}^{n}$ and $y \in \operatorname{span}\left\{e_{k}\right\}_{k>n}$, then

$$
\|x+y\|_{1} \leqslant\left(\|x\|_{1}^{2}+2\|y\|_{1}^{2}\right)^{1 / 2} .
$$

It follows that in the space $J$ considered with the norm $\|\cdot\|_{1}$ we have $\delta(t ; x) \leqslant(1$ $\left.+2 t^{2}\right)^{1 / 2}-1$ for all $x \in J$ with $\|x\|_{1}=1$ and $t \geqslant 0$.

In the next section we give an application of asymptotical smoothness to fixed point theory. Our main tool will be the following lemma.

Lemma 2.3. Let $X$ be an asymptotically smooth Banach space, $\left(x_{\alpha}\right)_{\alpha \in A}$ be a bounded net in $X$ and $x, y$ be weak limit points of subnets of $\left(x_{\alpha}\right)_{\alpha \in A}$. If the limit

$$
\lim _{\alpha \in A}\left\|t x_{\alpha}+(1-t) x-y\right\|
$$

exists for each $t \in[0,1]$, then $x=y$.

Proof: It suffices to consider the case when $y=0$. Suppose, contrary to our claim, that $x \neq 0$ and put $u=x /\|x\|, u_{\alpha}=x_{\alpha} /\|x\|$ and

$$
h(t)=\lim _{\alpha \in A}\left\|t u_{\alpha}+(1-t) u\right\| .
$$

Since $u$ is a weak cluster point of $\left(u_{\alpha}\right)_{\alpha \in A}, 1=\|u\| \leqslant h(t)$ for every $t \in[0,1]$. But $\left(u_{\alpha}\right)_{\alpha \in A}$ has a weakly null subnet. From Theorem 2.2 we therefore see that

$$
1 \leqslant h(t) \leqslant(1-t)\left(\delta\left(\frac{c t}{1-t} ; u\right)+1\right)
$$

for every $t \in[0,1)$, where $c=\sup _{\alpha \in A}\left\|u_{\alpha}\right\|$. Hence

$$
\frac{t}{1-t} \leqslant \delta\left(\frac{c t}{1-t} ; u\right)
$$

which contradicts the assumption that $X$ is asymptotically smooth.

The idea of Lemma 2.3 goes back to a result due to J. García Falset [7], where it was proved only for sequences under the assumption that $X$ is uniformly convex and its dual has the Kadec-Klee property (see also [17]). In [9], the García Falset lemma was generalised for nets in reflexive Banach spaces whose duals have the Kadec-Klee property. We shall show that this property is strongly related to asymptotical smoothness. Let us 
first establish terminology concerning the Kadec-Klee property. It/coincides with that of [4].

A Banach space $X$ has the Kadec property provided that if $\left(x_{\alpha}\right)_{\alpha \in A}$ is a net in $S_{X}$ converging weakly to $x \in S_{X}$, then $\left(x_{\alpha}\right)_{\alpha \in A}$ converges to $x$ in norm. Restricting this definition to sequences, we obtain the definition of the Kadec-Klee property. Next, replacing $X$ by $X^{*}$ and weak convergence by weak* convergence, we get the definitions of the $w^{*}$ Kadec and the $w^{*}$ Kadec-Klee property. Obviously, the Kadec property means that the restriction of the weak topology to $S_{X}$ coincides with the norm topology. A characterisation of the Kadec property in terms of a geometric modulus can be found in [19].

TheOREM 2.4. Let $X$ be a Banach space.

(i) If $X^{*}$ is asymptotically smooth, then $X$ has the Kadec property.

(ii) If $X^{*}$ has the $w^{*}$ Kadec property, then $X$ is asymptotically smooth.

Proof: Assume first that $X^{*}$ is asymptotically smooth and $X$ does not have the Kadec property. Then there exist a net $\left(x_{\alpha}\right)_{\alpha \in A}$ in $S_{X}$ and $\varepsilon>0$ such that $\left(x_{\alpha}\right)_{\alpha \in A}$ converges weakly to $x \in S_{X}$ and $\left\|x_{\alpha}-x\right\|>\varepsilon$ for every $\alpha$. We find $x^{*} \in S_{X^{*}}$ with $x^{*}(x)=1$ and a net $\left(x_{\alpha}^{*}\right)_{\alpha \in A}$ in $S_{X^{*}}$ such that $x_{\alpha}^{*}\left(x_{\alpha}-x\right)>\varepsilon$ for every $\alpha$. Then

$$
\begin{aligned}
\left\|x^{*}+t\left(x_{\alpha}^{*}-x_{\beta}^{*}\right)\right\| & \geqslant x^{*}\left(x_{\alpha}\right)+t x_{\alpha}^{*}\left(x_{\alpha}-x\right)-t x_{\beta}^{*}\left(x_{\alpha}-x\right)+t x_{\alpha}^{*}(x)-t x_{\beta}^{*}(x) \\
& \geqslant x^{*}\left(x_{\alpha}\right)+t \varepsilon-t x_{\beta}^{*}\left(x_{\alpha}-x\right)+t x_{\alpha}^{*}(x)-t x_{\beta}^{*}(x)
\end{aligned}
$$

for every $t \geqslant 0$ and all $\alpha, \beta$. Since, passing to a subnet if necessary, we can assume that $\lim _{\alpha \in A} x_{\alpha}^{*}(x)$ exists, it follows that

$$
1+\varepsilon t=x^{*}(x)+\varepsilon t \leqslant \liminf _{\beta \in A} \liminf _{\alpha \in A}\left\|x^{*}+t\left(x_{\alpha}^{*}-x_{\beta}^{*}\right)\right\| \leqslant \delta_{1}\left(t ; x^{*}\right)+1 .
$$

Here $\delta_{1}$ corresponds to the space $X^{*}$. In view of Theorem 2.2 this shows that $\varepsilon$ $\leqslant \delta\left(2 t ; x^{*}\right) / t$ for every $t>0$, which contradicts our assumption.

Assume now that $X^{*}$ has the $w^{*}$ Kadec property. We take $x \in S_{X}$, a weakly null net $\left(x_{\alpha}\right)_{\alpha \in A}$ in $B_{X}$ and $t>0$. For each $\alpha$ there is $x_{\alpha}^{*} \in S_{X^{*}}$ such that $x_{\alpha}^{*}\left(x+t x_{\alpha}\right)=\left\|x+t x_{\alpha}\right\|$. Passing to subnets, we can assume that $\lim _{\alpha \in A}\left\|x+t x_{\alpha}\right\|$ exists and $\left(x_{\alpha}^{*}\right)_{\alpha \in A}$ converges weak* to some $x^{*} \in B_{X^{*}}$. We have

$$
\begin{aligned}
\lim _{\alpha \in A}\left\|x+t x_{\alpha}\right\| & =\lim _{\alpha \in A} x_{\alpha}^{*}\left(x+t x_{\alpha}\right) \\
& =x^{*}(x)+t \lim _{\alpha \in A}\left(x_{\alpha}^{*}-x^{*}\right)\left(x_{\alpha}\right) \leqslant\left\|x^{*}\right\|+t \liminf _{\alpha \in A}\left\|x_{\alpha}^{*}-x^{*}\right\| .
\end{aligned}
$$

In view of Theorem 2.2 it is enough to show that for each $\varepsilon>0$ there exists $t>0$ such that

$$
\lim _{\alpha \in A}\left\|x+t x_{\alpha}\right\| \leqslant 1+\varepsilon t .
$$

To this end we put $a=\liminf _{\alpha \in A}\left\|x_{\alpha}^{*}-x^{*}\right\|$ and consider two cases. 
I. $a \leqslant \varepsilon$. Then (1) holds for every $t>0$.

II. $a>\varepsilon$. In this case by our assumption we have $\left\|x^{*}\right\|<1$ and it suffices to take $t=\left(1-\left\|x^{*}\right\|\right) /(a-\varepsilon)$.

We shall consider some examples related to Theorem 2.4. It is easy to see that the space $c$ of convergent sequences is not asymptotically smooth. The same is therefore true for $l_{\infty}$. On the other hand, $l_{1}$ has the Kadec property. This shows that the implication (i) can not be reversed and the $w^{*}$ Kadec property can not be replaced by the Kadec property in (ii).

Let now $\omega_{1}$ be the first uncountable ordinal. The space $C\left(\left[0, \omega_{1}\right]\right)$ admits an equivalent Fréchet differentiable norm $\|\cdot\|_{1}$ (see [25] or [6, p. 313]). By Proposition 2.1, $C\left(\left[0, \omega_{1}\right]\right)$ considered with this norm is asymptotically smooth. Given $\alpha \in\left[0, \omega_{1}\right]$, we put $f_{\alpha}(x)=x(\alpha)$ where $x \in C\left(\left[0, \omega_{1}\right]\right)$. Then there exist a constant $c>0$ and an increasing sequence $\left(\alpha_{n}\right)$ of ordinals in $\left[0, \omega_{1}\right)$ such that $\left\|f_{\alpha_{n}}\right\|_{1}=c$ for every $n$ and $\left\|f_{\alpha}\right\|_{1}=c$ where $\alpha=\lim _{n \rightarrow \infty} \alpha_{n}$ (see [25] or [6, p. 313]). Clearly, $f_{\alpha}$ is the weak ${ }^{*}$ limit of the sequence $\left(f_{\alpha_{n}}\right)$. It follows that $C\left(\left[0, \omega_{1}\right]\right)^{*}$ does not have the $w^{*}$ Kadec property. This shows that the implication opposite to (ii) is not true.

In some cases a property formulated in terms of sequences can be extended to nets. For instance, if a space $X$ is reflexive, then the Kadec-Klee property is equivalent to the Kadec property (see [1], p. 113 and [14]). Another result of this kind was obtained in [18]. Let us recall that a family $\left\{e_{t}\right\}_{t \in T}$ of vectors of a Banach space $X$ is called an M-basis of $X$ if there exist functionals $e_{t}^{*} \in X^{*}, t \in T$ such that

$$
e_{t}^{*}\left(e_{s}\right)= \begin{cases}0 & \text { if } t \in T \backslash\{s\} \\ 1 & \text { if } t=s\end{cases}
$$

and $\left\{e_{t}\right\}_{t \in T}$ is linearly dense in $X$. If in addition $\left\{e_{t}^{*}\right\}_{t \in T}$ is linearly dense in $X^{*}$, then the M-basis $\left\{e_{t}\right\}_{t \in T}$ is said to be shrinking. Given $x \in \operatorname{span}\left\{e_{t}\right\}_{t \in T}$, we put supp $x=\{t$ $\left.\in T: e_{t}^{*}(x) \neq 0\right\}$. Clearly, for any set $\Gamma$ the space $c_{0}(\Gamma)$ has a shrinking M-basis. Let us also point out that all reflexive spaces have shrinking M-bases (see [24, p. 716]). In [18], it was shown that if a Banach space $X$ has a shrinking M-basis, then

$$
\delta(t ; x)=\sup \left\{\inf _{n \in \mathbb{N}}\left\|x+t x_{n}\right\|-1\right\}
$$

for each $t \geqslant 0$ and each $x \in S_{X}$, where the supremum is taken over all weakly null sequences $\left(x_{n}\right)_{n \in \mathbb{N}}$ in $S_{X}$. It is easy to see that infimum can be replaced by limsup in this formula. Moreover, the supremum can be taken over all weakly null sequences $\left(x_{n}\right)_{n \in \mathbf{N}}$ in $B_{X}$ as well. This shows that for spaces with shrinking M-bases the formula in Theorem 2.2 can be reduced to sequences. We shall give a general method of establishing results of this kind. 
LEMMA 2.5. Let $\left(x_{\alpha}\right)_{\alpha \in A}$ be a bounded weakly null net in a Banach space $X$. If $X$ has a shrinking $M$-basis or the set $\left\{x_{\alpha}\right\}$ is relatively weakly compact, then there exists an increasing sequence $\left(\alpha_{n}\right)_{n \in \mathbb{N}}$ such that $\left(x_{\alpha_{n}}\right)_{n \in \mathbb{N}}$ converges weakly to zero.

Proof: Assume that $X$ has a shrinking M-basis $\left\{e_{t}\right\}_{t \in T}$. By induction we choose an increasing sequence $\left(\alpha_{n}\right)_{n \in \mathbb{N}}$ and a sequence $\left(y_{n}\right)_{n \in \mathbb{N}}$ in $Y=\operatorname{span}\left\{e_{t}\right\}$ in such a way that supp $y_{i} \cap \operatorname{supp} y_{j}=\emptyset$ if $i \neq j$ and $\left\|x_{\alpha_{n}}-y_{n}\right\|<1 / n$ for every $n$. To this end we take an arbitrary $\alpha_{1}$ and find $y_{1} \in Y$ for which $\left\|x_{\alpha_{1}}-y_{1}\right\|<1$. Next, having $\alpha_{1}, \ldots, \alpha_{n-1}$ and $y_{1}, \ldots, y_{n-1}$, we set $Z=\bigcup_{i=1}^{n-1} \operatorname{supp} y_{i}$ and $c=\left(1+\max _{t \in Z}\left\|e_{t}^{*}\right\|\right) \sum_{t \in Z}\left\|e_{t}\right\|$. There is $\beta$ such that if $\alpha \geqslant \beta$, then $\left|e_{t}^{*}\left(x_{\alpha}\right)\right|<1 /(2 c n)$ for every $t \in Z$. We take arbitrary $\alpha_{n} \geqslant \beta$ and find $y \in Y$ such that $\left\|x_{\alpha_{n}}-y\right\|<1 /(2 c n)$. Then $\left|e_{t}^{*}(y)\right|<\left(1+\left\|e_{t}^{*}\right\|\right) /(2 c n)$ for every $t \in Z$. We set $y_{n}=y-\sum_{t \in Z} e_{t}^{*}(y) e_{t}$. Clearly, supp $y_{n} \cap \operatorname{supp} y_{i}=\emptyset$ for $i=1, \ldots, n-1$ and

$$
\left\|x_{\alpha_{n}}-y_{n}\right\| \leqslant\left\|x_{\alpha_{n}}-y\right\|+\left\|y-y_{n}\right\|<\frac{1}{n} .
$$

Moreover, it is easy to see that $\left(y_{n}\right)_{n \in \mathbb{N}}$ converges weakly to zero and so does $\left(x_{\alpha_{n}}\right)_{n \in \mathbb{N}}$.

Consider now the case when $X$ is arbitrary and $\left\{x_{\alpha}\right\}$ is relatively weakly compact. If $\lim _{\alpha \in A}\left\|x_{\alpha}\right\|=0$, then we find an increasing sequence $\left(\alpha_{n}\right)_{n \in \mathbb{N}}$ so that $\lim _{n \rightarrow \infty}\left\|x_{\alpha_{n}}\right\|=0$. In the other case the proof hinges on the well known Mazur method of constructing basic sequences (see [3]). Since $\left(x_{\alpha}\right)_{\alpha \in A}$ does not converge to zero, passing to a subnet, we can assume that inf $f_{\alpha \in A}\left\|x_{\alpha}\right\|>0$. Then, using Mazur's technique, we find an increasing sequence $\left(\alpha_{n}\right)_{n \in \mathbb{N}}$ so that $\left(x_{\alpha_{n}}\right)_{n \in \mathbb{N}}$ is a basic sequence. Recall that induction is used in this technique, so having $\alpha_{1}, \ldots, \alpha_{n-1}$, we get $\beta$ such that $\alpha_{n}$ can be chosen as an arbitrary element of the set $\{\alpha \in A: \alpha \geqslant \beta\}$. The sequence $\left(x_{\alpha_{n}}\right)_{n \in \mathbb{N}}$ has a weakly convergent subsequence. Since it is a basic sequence, its weak limit equals zero.

REMARK 2.1. A result analogous to Lemma 2.5 holds for bounded weak* null nets $\left(x_{\alpha}^{*}\right)_{\alpha \in A}$ in $X^{*}$. Namely, if $X$ has a shrinking M-basis or the set $\left\{x_{\alpha}^{*}\right\}$ is relatively weak ${ }^{*}$ sequentially compact, then there exists an increasing sequence $\left(\alpha_{n}\right)_{n \in \mathbb{N}}$ such that $\left(x_{\alpha_{n}}^{*}\right)_{n \in \mathbb{N}}$ converges weak* to zero.

Using Lemma 2.5, we can extend the aforementioned result on the Kadec-Klee property given in [14].

Corollary 2.6. Let $X$ be a Banach space with the Kadec-Klee property and $\left(x_{\alpha}\right)_{\alpha \in A}$ be a net in $S_{X}$ weakly converging to $x \in S_{X}$. If $X$ has a shrinking $M$-basis or $\left\{x_{\alpha}\right\}$ is relatively weakly compact, then $\left(x_{\alpha}\right)_{\alpha \in A}$ converges to $x$ in norm.

Proof: Passing to a subnet, we can assume that the limit $c=\lim _{\alpha \in A}\left\|x_{\alpha}-x\right\|$ exists. Following the reasoning given in the proof of Lemma 2.5, one can find a sequence $\alpha_{1}$ $\leqslant \alpha_{2} \leqslant \ldots$ such that the sequence $\left(x_{\alpha_{n}}\right)_{n \in \mathbb{N}}$ converges weakly to $x$ and $\lim _{n \rightarrow \infty}\left\|x_{\alpha_{n}}-x\right\|=c$. By assumption, $c=0$, which concludes the proof. 
Corollary 2.6 gives us the first part of the following result. The second one can be obtained in a similar way.

Proposition 2.7. Let $X$ be a Banach space with a shrinking $M$-basis. Then

1. $X$ has the Kadec property if and only if $X$ has the Kadec-Klee property.

2. $X^{*}$ has the $w^{*}$ Kadec property if and only if $X^{*}$ has the $w^{*}$ Kadec-Klee property.

Combining Theorem 2.4 and Proposition 2.7 leads to the following corollary.

Corollary 2.8. Let $X$ be a reflexive space. Then $X$ is asymptotically smooth if and only if $X^{*}$ has the Kadec-Klee property.

Let us mention another property with many applications in metric fixed point theory. A Banach space $X$ has the Opial property if

$$
\limsup _{n \rightarrow \infty}\left\|x_{n}\right\|<\underset{n \rightarrow \infty}{\limsup }\left\|x_{n}-x\right\|
$$

whenever $\left(x_{n}\right)_{n \in \mathbb{N}}$ is a weakly null sequence in $X$ and $x \neq 0$. Some authors (see [13]) consider a condition obtained from the above definition by replacing sequences $\left(x_{n}\right)_{n \in \mathbb{N}}$ by nets. A reasoning similar to that in the proof of Corollary 2.6 yields the following result.

Proposition 2.9. Let $X$ be a Banach space with the Opial property, $\left(x_{\alpha}\right)_{\alpha \in A}$ be a weakly null net in $X$ and $x \in X, x \neq 0$. If $X$ has a shrinking $M$-basis or $\left\{x_{\alpha}\right\}$ is relatively weakly compact, then

$$
\underset{\alpha \in A}{\limsup }\left\|x_{\alpha}\right\|<\limsup _{\alpha \in A}\left\|x_{\alpha}-x\right\| .
$$

\section{WEAK CONVERGENCE OF ALMOST ORBITS}

In this section we give some results about weak convergence of almost orbits of three different types of semigroups. We start with some definitions and notations.

Throughout this section $C$ denotes a weakly compact subset of a Banach space $X$. Let $G$ be a semitopological semigroup, that is, $G$ is a semigroup with a Hausdorff topology such that for each $s \in G$ the mappings $s \mapsto t \cdot s$ and $s \mapsto s \cdot t$ from $G$ to $G$ are continuous. $G$ is said to be right reversible if any two closed left ideals of $G$ have nonvoid intersection. In this case $(G, \leqslant)$ is a directed system where the binary relation " $\leqslant$ " on $G$ is defined by: $s \leqslant t$ if and only if $\{s\} \cup \overline{G s} \supset\{t\} \cup \overline{G t}$ for $s, t \in G$. Now let $S=\{T(t): t \in G\}$ be a family of self-mappings of $C$.

Then $S$ is said to be an asymptotically nonexpansive-type semigroup on $C$ if the following conditions are satisfied:

(i) the mapping $(s, x) \mapsto T(s) x$ from $G \times C$ into $C$ is continuous on $G \times C$ furnished with the product topology, 
(ii) $T(t s) x=T(t) T(s) x$ for all $t, s \in G$ and $x \in C$,

(iii) for each $t \in G$ and $x \in C$ there exists $r(t, x)>0$ such that

$$
\|T(t) x-T(t) y\| \leqslant\|x-y\|+r(t, x)
$$

for all $y \in C$ with

$$
\lim _{t \in G} r(t, x)=0
$$

If the third assumption is replaced by

(iii') there exists a net $\left(k_{t}\right)_{t \in G}$ of positive numbers such that

$$
\lim _{t \in G} k_{t}=1
$$

and

$$
\|T(t) x-T(t) y\| \leqslant k_{t}\|x-y\|
$$

for all $t \in G$ and $x, y \in C$,

then $S$ is said to be an asymptotically nonexpansive semigroup on $C$.

If in (iii') $k_{t}=1$ for $t \in G$, we simply say that $S$ is a nonexpansive semigroup.

We denote by $\operatorname{Fix}(S)$ the set of all common fixed points of $S$, that is, $\operatorname{Fix}(S)$ $=\bigcap_{t \in G} \operatorname{Fix}(T(t))$.

We say that a function $u(\cdot): G \rightarrow C$ is an almost orbit of the semigroup $S$ if

$$
\lim _{t \in G}\left[\sup _{h \in G}\|u(h t)-T(h) u(t)\|\right]=0 .
$$

If $u$ is an almost orbit of the semigroup $S$, then $\omega_{w}(u)$ denote the set of all weak limit points of subnets of the net $(u(t))_{t \in G}$.

To prove our next result we need the following three lemmas. The first two of them are due to $\mathrm{Li}[\mathbf{1 5}]$.

LEMMA 3.1. Let $X$ be a uniformly convex Banach space and $C$ be a nonempty bounded closed convex subset of $X$. Let $S=\{T(t): t \in G\}$ be a right reversible semitopological semigroup of asymptotically nonexpansive-type on $C$ with nonempty $\operatorname{Fix}(S)$. If $u(\cdot)$ is an almost orbit of $S$ and $f \in \operatorname{Fix}(S)$, then $\lim _{t \in G}\|u(t)-f\|$ exists.

LEMMA 3.2. Let $X$ be a uniformly convex Banach space and $C$ be a nonempty bounded closed convex subset of $X$. Let $S=\{T(t): t \in G\}$ be a right reversible semitopological semigroup of asymptotically nonexpansive-type on $C$ with nonempty $\operatorname{Fix}(S)$. Suppose that $0<\lambda<1$ and $f \in \operatorname{Fix}(S)$. Then for any $\varepsilon>0$ there exists $s_{0}=s_{0}(\lambda, f, \varepsilon) \in G$ such that

$$
\underset{t \in G}{\limsup }\|T(t)(\lambda u(s)+(1-\lambda) f)-(\lambda T(t) u(s)+(1-\lambda) f)\|<\varepsilon
$$

for all $s \geqslant s_{0}$. 
The above lemma allows us to prove the next one.

Lemma 3.3. Let $X$ be a uniformly convex Banach space and $C$ be a nonempty bounded closed convex subset of $X$. Let $S=\{T(t): t \in G\}$ be a right reversible semitopological semigroup of asymptotically nonexpansive-type on $C$ with nonempty $\operatorname{Fix}(S)$. If $u(\cdot)$ is an almost orbit of $S, 0<\lambda<1$ and $f, g \in \operatorname{Fix}(S)$, then

$$
\lim _{t \in G}\|\lambda u(t)+(1-\lambda) f-g\|
$$

exists.

Proof: Take $\varepsilon>0$. By Lemma 3.2 there exists $s_{0} \in G$ such that

$$
\limsup _{t \in G}\|T(t)(\lambda u(s)+(1-\lambda) f)-(\lambda T(t) u(s)+(1-\lambda) f)\|<\varepsilon
$$

for all $s \geqslant s_{0}$. Hence we get

$$
\begin{aligned}
\|\lambda u(t s)+(1-\lambda) f-g\| \leqslant & \|\lambda u(t s)-\lambda T(t) u(s)\| \\
& +\|T(t)(\lambda u(s)+(1-\lambda) f)-(\lambda T(t) u(s)+(1-\lambda) f)\| \\
& +\|T(t)(\lambda u(s)+(1-\lambda) f)-g\| \\
\leqslant & \lambda \sup _{h \in G}\|u(h s)-T(h) u(s)\| \\
& +\|\lambda T(t) u(s)+(1-\lambda) f-T(t)(\lambda u(s)+(1-\lambda) f)\| \\
& +r(t, g)+\|\lambda u(s)+(1-\lambda) f-g\| .
\end{aligned}
$$

Therefore we have

$\limsup _{t \in G}\|\lambda u(t)+(1-\lambda) f-g\| \leqslant \sup _{h \in G}\|u(h s)-T(h) u(s)\|+\varepsilon+\|\lambda u(s)+(1-\lambda) f-g\|$, and finally,

$$
\limsup _{t \in G}\|\lambda u(t)+(1-\lambda) f-g\| \leqslant \varepsilon+\liminf _{s \in G}\|\lambda u(s)+(1-\lambda) f-g\| .
$$

Since $\varepsilon>0$ is arbitrary, we obtain

$$
\limsup _{t \in G}\|\lambda u(t)+(1-\lambda) f-g\| \leqslant \liminf _{s \in G}\|\lambda u(s)+(1-\lambda) f-g\|,
$$

which concludes the proof.

Using the above lemmas we can prove the following theorem.

TheOREM 3.4. Let $X$ be a uniformly convex and asymptotically smooth Banach space, and let $C$ be a nonempty bounded closed convex subset of $X$. If $S=\{T(t): t \in G\}$ is a right reversible semitopological semigroup of asymptotically nonexpansive-type on $C$ with nonempty $\operatorname{Fix}(S)$ and $u(\cdot)$ is an almost orbit of $S$, then the following statements are equivalent: 
(1) $w-\lim _{t \in G} u(t)=p \in \operatorname{Fix}(S)$;

(2) $\omega_{w}(u) \subset \operatorname{Fix}(S)$.

ProOF: We only need to show that $(2) \Longrightarrow(1)$. Taking $f, g \in \omega_{w}(u)$ we get $f, g \in \operatorname{Fix}(S)$. By Lemmas 3.1 and 3.3 this implies that

$$
\lim _{t \in G}\|\lambda u(t)+(1-\lambda) f-g\|
$$

exists for each $0 \leqslant \lambda \leqslant 1$. To end the proof it suffices to apply Lemma 2.3 and Theorem 2.4 .

It is known that each commutative semigroup $G$ is right reversible. In this case we use the standard notation, that is, we replace the operation - by + . Then the order is simply defined in the following way: $s \leqslant t$ if and only if there is $q \in G$ with $s+q=t$ and we additionally assume that $0 \in G$ and $T(0) x=x$ for all $x \in C$.

For asymptotically nonexpansive semigroups we first recall the following result due to Oka [20].

THEOREM 3.5. Let $X$ be a uniformly convex Banach space and $C$ be a nonempty bounded closed convex subset of $X$. Let $S=\{T(t): t \in G\}$ (where $G$ is a commutative semigroup with an identity) be an asymptotically nonexpansive semigroup on $C$. If $u$ is an almost orbit of $S$ and

$$
w-\lim _{t \in G}(u(t+h)-u(t))=0
$$

for each $h \in G$, then

$$
\omega_{w}(u) \subset \operatorname{Fix}(S)
$$

Now we rewrite the result from [9] in terms of asymtotical smoothness and to make the paper self-contained we also present its proof.

THEOREM 3.6. Let $X$ be a uniformly convex and asymptotically smooth Banach space and $C$ be its nonempty bounded closed convex subset. Let $S=\{T(t): t \in G\}$ (where $G$ is a commutative semigroup with an identity) be an asymptotically nonexpansive semigroup on $C$. If $u(\cdot)$ is an almost orbit of $S$, then the following statements are equivalent:

(1) $w-\lim _{t \in G} u(t)=p \in \operatorname{Fix}(S)$;

(2) $\omega_{w}(u) \subset \operatorname{Fix}(S)$;

(3) $w-\lim _{t \in G}(u(t+h)-u(t))=0$ for every $h \in G$, that is, $u$ is weakly asymptotically regular.

Proof: By Theorem 3.4, (1) and (2) are equivalent. It follows from the above theorem that $(3) \Longrightarrow(2)$. The implication (1) $\Longrightarrow(3)$ is obvious.

In [13] and [16] the authors use the Opial property for nets. In view of Proposition 2.9 we can reformulate the first result in the following way. 
THEOREM 3.7. Suppose that $X$ has the Opial property. Let $C$ be a weakly compact subset of $X, G$ be a right reversible semitopological semigroup and $S=\{T(t): t$ $\in G\}$ be a nonexpansive semigroup on $C$. If $u(\cdot)$ is an almost orbit of $S$, then $\{u(t): t$ $\in G\}$ is weakly convergent (to a fixed point) if and only if it is weakly asymptotically regular.

The second result is about commutative semigroups. Recall that a Banach space $X$ has the uniform Kadec-Klee (UKK for short) property whenever for any $\varepsilon>0$ there is $\delta>0$ such that if $x$ is a weak limit of a sequence $\left(x_{n}\right)_{n \in \mathbb{N}}$ in $B_{X}$ with $\inf \left\{\left\|x_{n}-x_{m}\right\|: n\right.$ $\neq m\}>\varepsilon$, then $\|x\| \leqslant 1-\delta$.

THEOREM 3.8. Suppose that $X$ has the Opial property and the norm of $X$ is UKK. Let $C$ be a weakly compact and convex subset of $X, S=\{T(t): t \in G\}$ be a commutative semigroup of asymptotically nonexpansive-type on $C$, and let $u(\cdot)$ be an almost orbit of $S$. Then $\{u(t): t \in G\}$ is weakly convergent (to a fixed point) if and only if it is weakly asymptotically regular.

\section{REFERENCES}

[1] A.G. Aksoy and M.A. Khamsi, Nonstandard methods in fixed point theory (Springer-Verlag, New York, 1990).

[2] S. Banach, Euvres, Éditions Scientifiques de Pologne, vol. II (PWN, Warsaw, 1979).

[3] C. Bessaga and A. Pelczyński, 'On bases and unconditional convergence of series in Banach spaces', Studia Math. 17 (1958), 151-164.

[4] J.M.F. Castillo and M. González, Three space problem in Banach space theory, Lecture Notes in Mathematics 1667 (Springer-Verlag, Berlin, 1997).

[5] M.M. Day, Normed linear spaces (Springer-Verlag, Berlin, Göttingen, Heidelberg, 1962).

[6] R. Deville, G. Godefroy and V. Zizler, Smoothness and renormings in Banach spaces, Pitman Monographs and Surveys in Pure and Applied Mathematics 64 (Longman Scientific and Technical, Harlow, 1993).

[7] J. García Falset, W. Kaczor, T. Kuczumow and S. Reich, 'Weak convergence theorems for asymptotically nonexpansive mappings and semigroups', Nonlinear Anal. 43 (2001), $377-401$.

[8] R.C. James, 'A non-reflexive Banach space isometric to its second conjugate', Proc. Nat. Acad. Sci U.S.A. 37 (1951), 174-177.

[9] W. Kaczor, 'Weak convergence of almost orbits of asymptotically nonexpansive commutative semigroups', (preprint).

[10] W. Kaczor, T. Kuczumow and S. Reich, 'A mean ergodic theorem for nonlinear semigroups which are asymptotically nonexpansive in the intermediate sense', J. Math. Anal. Appl. 246 (2000), 1-27.

[11] W. Kaczor, T. Kuczumow and S. Reich, 'A mean ergodic theorem for mappings which are asymptotically nonexpansive in the intermediate sense', Nonlinear Anal. 47 (2001), 2731-2742.

[12] W. Kaczor and T. Sẹkowski, 'Weak convergence of iteration process for nonexpansive mappings and nonexpansive semigroups', Ann. Univ. Mariae Curie-Sktodowska, Sect. A 52 (1998), 71-77. 
[13] J.K. Kim and G. Li, 'Asymptotic behaviour for an almost-orbit of nonexpansive semigroups in Banach spaces', Bull. Austral. Math. Soc. 61 (2000), 345-350.

[14] W.A. Kirk, 'An iteration process for nonexpansive mappings with applications to fixed point theory in product spaces', Proc. Amer. Math. Soc. 107 (1989), 411-415.

[15] G. Li, 'Weak convergence and non-linear ergodic theorems for reversible semigroups of non-lipschitzian mappings', J. Math. Anal. Appl. 206 (1997), 451-464.

[16] G. Li, 'Asymptotic behavior for commutative semigroups of asymptotically nonexpansive-type mappings', Nonlinear Anal. 42 (2000), 175-183.

[17] G. Li and B. Sims, 'Ergodic theorem and strong convergence of averaged approximations for non-lipschitzian mappings in Banach spaces', (preprint).

[18] E. Maluta, S. Prus and M. Szczepanik, 'On Milman's moduli for Banach spaces', Abst. Appl. Anal. 6 (2001), 115-129.

[19] V.D. Milman, 'Geometric theory of Banach spaces. Part II, Geometry of the unit sphere', (Russian), Usp. Mat. Nauk. 26 (1971), 73-149. English translation: Russian Math. Surv. 26 (1971), 79-163.

[20] H. Oka, 'Nonlinear ergodic theorems for commutative semigroups of asymptotically nonexpansive mappings', Nonlinear Anal. 7 (1992), 619-635.

[21] Z. Opial, 'Weak convergence of the sequence of successive approximations for nonexpansive mappings', Bull. Amer. Math. Soc. 73 (1967), 591-597.

[22] S. Prus, 'On infinite dimensional uniform smoothness of Banach spaces', Comment. Math. Univ. Carolinae 40 (1999), 97-105.

[23] S. Prus, 'Geometrical background of metric fixed point theory', in Handbook of Metric Fixed Point Theory, (W. A. Kirk and B. Sims, Editors) (Kluwer Academic Publishers, Dordrecht, 2001), pp. 93-132.

[24] I. Singer, Bases in Banach spaces II (Springer-Verlag, Berlin, 1981).

[25] M. Talagrand, 'Renormages de quelques $C(K)$ ', Israel J. Math. 54 (1986), 327-334.

\author{
Instytut Matematyki \\ University M. Curie-Sklodowska \\ 20-031 Lublin \\ Poland \\ e-mail: wkaczor@golem.umcs.lublin.pl \\ bsprus@golem.umcs.lublin.pl
}

